\title{
Central nervous system lesions in von Hippel-Lindau syndrome
}

H P H Neumann, H R Eggert, R Scheremet, M Schumacher, M Mohadjer, A K Wakhloo, B Volk, U Hettmannsperger, P Riegler, P Schollmeyer, O Wiestler

\begin{abstract}
CNS manifestations were studied in 97 gene carriers of von Hippel-Lindau syndrome (HLS). Haemangioblastomas of the CNS were found in 43 patients $(44 \%)$, 23 females and 20 males. The mean age at diagnosis was 39 years (12-73 years). A total of 93 haemangioblastomas were detected of which $74 \%$ were intracranial and $26 \%$ were located in the spinal cord; $75 \%$ were predominantly cystic and $25 \%$ presented as solid lesions. Multiple lesions were found in $42 \%$ of HLS-associated haemangioblastomas, but in none of 51 patients with CNS haemangioblastoma without HLS. Haemangioblastoma was the cause of death in $82 \%$ of patients with HLS. Although microsurgery has considerably improved post-operative results, multifocal tumour development and recurrence remain a serious problem in the clinical management of HLS gene carriers.
\end{abstract}

$(\Im$ Neurol Neurosurg Psychiatry 1992;55:898-901)

Albert-LudwigsUniversity, Freiburg, Germany Department of Medicine H P H Neumann P Schollmeyer

Department of Neurosurgery H R Eggert R Scheremet M Mohadjer

Department of Neuroradiology M Schumacher A K Wakhloo

Department of

Neuropathology B Volk

U Hettmannsperger

Ospedale Generale, Bolzano, Italy Department of Nephrology

P Riegler

University of Zürich, Zürich, Switzerland Department of Pathology $\mathrm{O}$ Wiestler

Correspondence to: Dr Neumann, Medizinische Klinik, Hugstetter Str 55, D-7800 Freiburg, Germany

Received 7 October 1991 and in revised form 6 January 1992.

Accepted 13 January 1992
Von Hippel-Lindau syndrome (HLS) is a familial disorder with an autosomal dominant pattern of inheritance. Afflicted patients develop a variety of tumours in different organs. Common lesions are angiomatosis of the retina, CNS haemangioblastomas, renal cysts and cancer, pancreatic cysts, phaeochromocytoma and epididymal cystadenoma. ${ }^{12}$ Although eponymous and a frequent lesion associated with the syndrome, Lindau's tumour, ${ }^{3}$ - that is, cystic haemangioblastoma of the cerebellum, is not found in every individual afflicted, nor is it the only neuropathological lesion in HLS patients. We have studied a large series of HLS patients to evaluate the prevalence and location of CNS lesions, together with the morbidity, mortality and occurrence of lesions associated with HLS in other organs.

Table 1 Screening program for potential HLS gene carriers

Family history and pedigree analysis ${ }^{\star}$

Blood pressure, catecholamine assay in plasma or 24 hour urine

Ophthalmological examination

Neurological examination

Cranial MRI using gadolinium enhancement

CT and ultrasound ${ }^{\star}$ scanning of the abdomen Ultrasound assessment of the testes

*Basic screening program for children from 6 to 10 years. Follow-up screening every 12-24 months recommended.

\section{Methods}

This study included 97 patients who were confirmed gene carriers for von Hippel-Lindau syndrome. Carriers were identified as being affected members of known HLS kindreds or by detecting additional manifestations of the syndrome, or both. ${ }^{12}$

Since 1976, all patients presenting with CNS haemangioblastoma at our institute were systematically registered and examined. For these patients, a screening programme (table 1) for HLS was developed and used for a prospective study. All first-degree relatives of afflicted individuals were included. For this purpose, an extensive pedigree analysis was performed, and patients with CNS lesions treated at other hospitals were also entered in the study.

CNS lesions were confirmed histopathologically using biopsy or necropsy material, by conventional staining methods. Immunohistochemical staining techniques were used in selected cases. The few CNS lesions that had not been surgically investigated, were classified as haemangioblastomas because of a history of previous haemangioblastoma, multifocal manifestations, a characteristic appearance in gadolinium-enhanced MRI and a typical location in the posterior cranial fossa or spinal canal.

Diagnostic imaging has been by CT and angiography since $1976, \mathrm{MRI}$ since 1987 , and contrast-enhanced MRI (using gadoliniumDTPA) since 1989.

\section{Results}

CNS HAEMANGIOBLASTOMA

CNS haemangioblastomas were found in 43 gene carriers from 30 families. There were 23 females and 20 males; the age at diagnosis ranged from 12-60 years (average 33.9). Of the 21 patients still alive, 15 are female, 6 are male, with an age range of 19-73 years (average 40 ). Of the 22 patients who died, 9 were female, 13 were male, with an age range of 12-60 years (average 39 ). In $82 \%$ of these patients, haemangioblastoma was the cause of death. After 1980, however, only one of 14 HLS carriers and none of 43 with sporadic haemangioblastomas died because of CNS haemangioblastoma. This suggests that modern neuroimaging techniques and microsurgery have considerably improved the prognosis of these patients.

The total number of haemangioblastomas observed in these patients was 93, of which $73 \%$ were located in the posterior fossa, $26 \%$ in the spinal canal, and $1 \%$ was supratentorial 
Table 2 Occurrence and location of CNS lesions in forty individuals with von Hippel-Lindau syndrome

\begin{tabular}{|c|c|c|c|c|c|c|c|c|}
\hline \multirow[b]{2}{*}{ Tumour } & \multicolumn{2}{|c|}{ Occurrence* } & \multicolumn{2}{|c|}{ Posterior fossa } & \multicolumn{2}{|c|}{ Supratentorial } & \multicolumn{2}{|c|}{ Spinal canal } \\
\hline & Patients & Tumours & Patients & Tumours & Patients & Tumours & Patients & Tumours \\
\hline $\begin{array}{l}\text { Haemangioblastoma } \\
\text { Metastatic carcinoma }\end{array}$ & $\begin{array}{r}43 \\
2\end{array}$ & $\begin{array}{r}93 \\
3\end{array}$ & $\begin{array}{r}39 \\
1\end{array}$ & $\begin{array}{r}68 \\
1\end{array}$ & $\begin{array}{l}1 \\
1\end{array}$ & $\begin{array}{l}1 \\
1\end{array}$ & $\begin{array}{l}7 \\
1\end{array}$ & $\begin{array}{r}24 \\
1\end{array}$ \\
\hline Total & 45 & 96 & 40 & 69 & 2 & 2 & 8 & 25 \\
\hline
\end{tabular}

${ }^{\star}$ Multifocal lesions occurred in 18 patients.

(table 2). Sixty seven haemangioblastomas were identified by biopsy, nine were found at necropsy, and 17 were diagnosed by neuroradiology. Seventy-five per cent of the tumours were cystic and $25 \%$ were solid; in the posterior fossa, $86 \%$ were cystic and in the spine, $46 \%$. The supratentorial lesion was solid.

Histopathology showed characteristic haemangioblastomas (World Health Organisation grade I) in all cases. No significant difference in histopathological appearance was noted between sporadic haemangioblastomas and those associated with HLS.

Multifocal haemangioblastomas occurred in 18 patients ( $42 \%$ ); of these, eight have died. At the time of the original diagnosis, multiple lesions were found in nine $(21 \%)$. Five patients

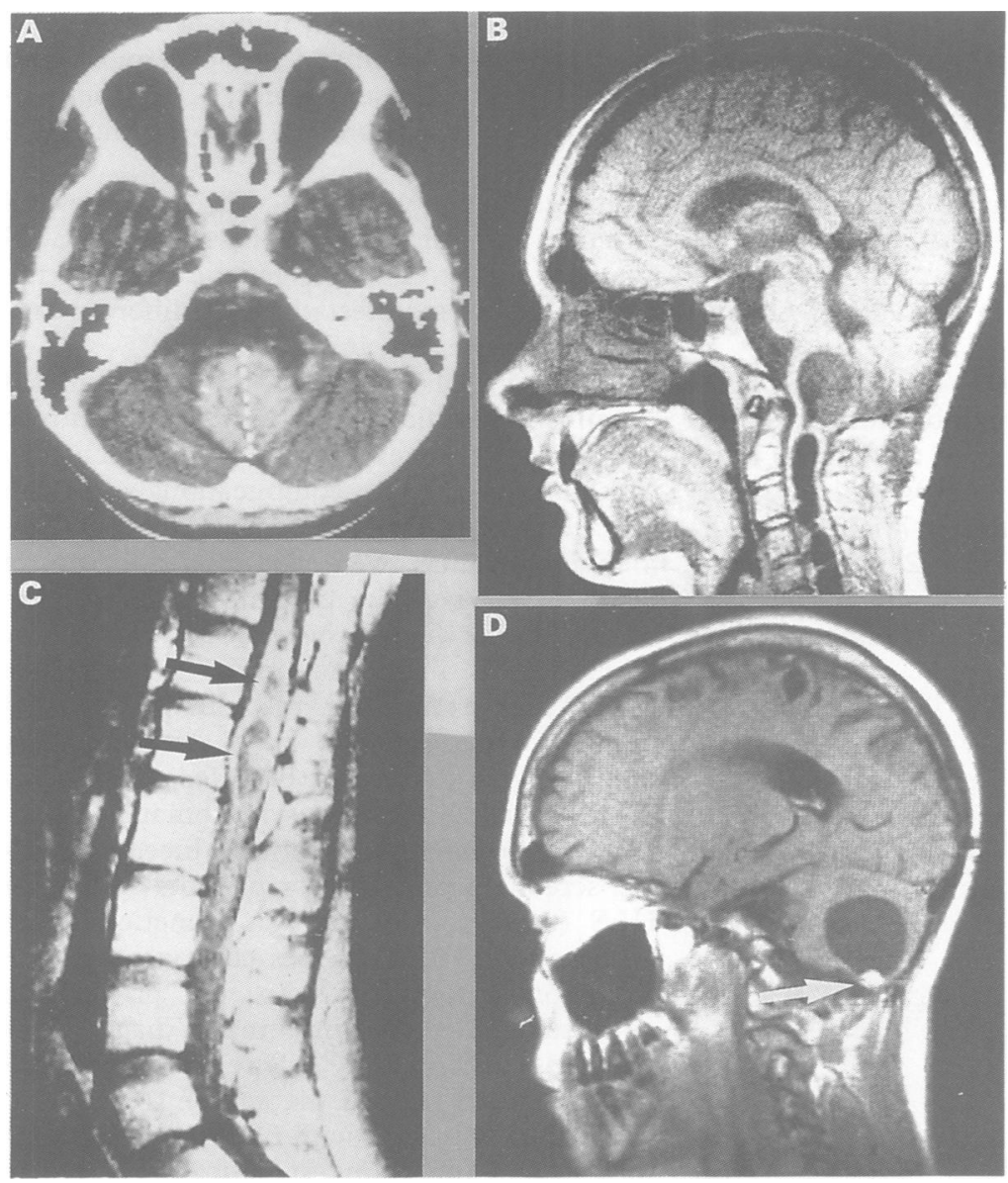

Figure 1 Multifocal CNS haemangioblastoma which required repeated neurosurgical interventions in a 20 year old woman patient with HLS. (a) Initial CT scan, 1982. Cerebellar haemangioblastoma. (b) MRI scan, 1987. Cystic medullary and spinal haemangioblastomas (T1 weighted images, 500/20). (c) MRI scan (proton density 2000/70), 1988. Multiple haemangioblastomas (arrows) of the thoracic and lumbar spinal cord (courtesy of Dr Fürmaier, Freiburg). (d) MRI scan, gadolinium-enhanced, T1 image (500/20), 1989. Cerebellar haemangioblastoma with solid tumour nodule (arrow) and a large cystic portion. with haemangioblastoma had surgery twice; two, three times; one, four times, and one, six times (fig 1).

In contrast, none of 51 patients (mean age 47 years, 18 female, 33 male) presenting with a sporadic CNS haemangioblastoma during the last 13 years developed multiple lesions; all these subjects underwent screening for HLS according to the programme in table 1 . Their mean age was $47 \cdot 2$ years and is significantly different $(p<0.05)$ from the mean age of the HLS carriers (33.9) years.

Tumours were surgically removed when signs of increased intracranial pressure or focal neurological deficits occurred or neuroradiological examination revealed evidence for rapid growth. When MRI scans showed lesions compatible with CNS haemangioblastoma in 14 patients, clinical follow-up revealed no signs of tumour progression. Surgery has not been performed on these patients so far.

Post-operative gadolinium-enhanced MRI was conducted annually in cases with a solitary tumour, and at six-monthly intervals in patients with multifocal lesions. Since the introduction of microsurgery post-operative outcome has been generally good. Of 19 patients, one died of multilocular haemangioblastomas, one had residual paraparesis and one had quadriplegia caused by spinal lesions. The other patients recovered completely.

Polycythaemia, a well-documented feature in CNS haemangioblastoma was present in only five of 43 cases (12\%).

\section{Metastases}

Metastatic involvement of the CNS occurred in two HLS patients. The first was a 42 year old woman with a history of a renal cell carcinoma. Two years after nephrectomy this patient experienced the symptoms of Brown-Séquard syndrome, caused by a mass in the D4-region (fig 2a). Histopathology disclosed metastatic renal cell cancer. To distinguish between clear cell carcinoma and haemangioblastoma, immunocytochemical reactions were carried out with antibodies to the pan-epithelial antigen Lu-5, a marker for cells of epithelial origin which is not expressed in haemangioblastomas. This reaction was strongly positive, confirming the diagnosis of metastatic carcinoma (fig 2b). The second was a 60 year old woman with bilateral retinal angiomatosis and a long history of maldigestion and malabsorption who developed severe pneumonia and died. Major findings at necropsy included isletcell carcinoma of the pancreas with metastases to the liver, the cerebellum and the dura mater, and renal cell cancer. 


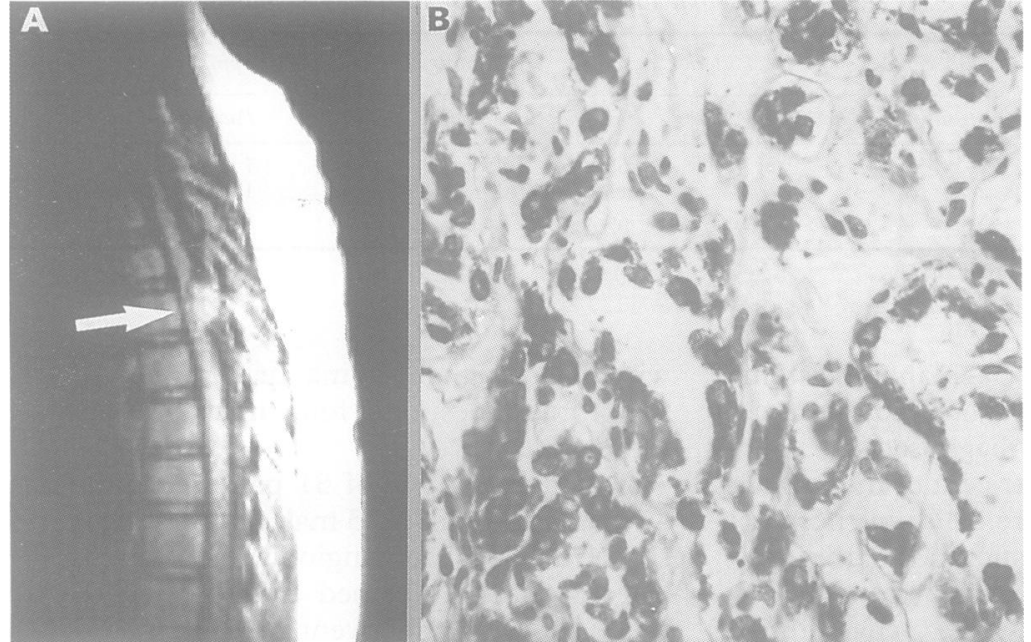

Figure 2 Metastatic renal cell carcinoma of the spinal cord in a 42 year old HLS patient. (a) MRI scan. Solid tumor (arrow) of the thoracic spinal cord

(Gadolinium-enhanced T1 weighted image, 500/20). (b) Histopathological appearance with features of a clear cell carcinoma. The epithelial nature of this neoplasm was confirmed using an immunocytochemical reaction for the pan-epithelial antigen Lu-5, a marker for cells of epithelial origin which is not found in haemangioblastomas. $(\times 250)$.

Differential diagnosis of CNS haemangioblastoma and metastatic renal cell carcinoma

The distinction between CNS haemangioblastoma and metastatic renal cell carcinoma can pose a major histopathological problem. To differentiate these two neoplasms, reticulin impregnation and antibodies to keratinassociated antigens were used. We have encountered three cases which, on the basis of retrospective histopathological and immunocytochemical analyses, had to be reclassified (fig 3 ).

Incidence of CNS lesions in von Hippel-Lindau syndrome

In the present study, $44 \%$ of 97 HLS gene carriers had CNS haemangioblastomas. Other HLS manifestations occurred as follows: retinal angiomatosis in $44 \%$ (19 of 43), renal cysts, renal cancer or both in $47 \%$ (20 of 43 ), phaeochromocytoma in $5 \%$ (2 of 43 ), pancreatic cysts in $30 \%$ (13 of 43 ) and epididymal cystadenoma in $15 \%$ ( 3 of 20 ). Nine patients

Figure 3

Histopathological appearance of a cerebellar haemangioblastoma in a 60 year old female. Because of its clear cell morphology, this tumor was initially misdiagnosed as metastatic renal cell carcinoma. The diagnosis of haemangioblastoma was strongly supported by the lack of expression of the pan-epithelial antigen Lu-5. (Stain: $H$ and $E$, $\times 250$ ).
(21\%) with CNS haemangioblastoma did not present with additional manifestations of the syndrome at the time of initial diagnosis.

In $56 \%$ of HLS gene carriers, no CNS haemangioblastoma was found. However, only $74 \%$ of these patients were examined by cranial CT, MRI or postmortem neuropathological analysis; the remaining patients had a normal neurological status. In seven affected families, CNS haemangioblastoma was not found among the features of the syndrome.

\section{Discussion}

The von Hippel-Lindau syndrome is an autosomal dominant inherited disorder in which sufferers are prone to develop cancer. Recent data indicate that HLS is more common than previously anticipated. The prefvalence is in the range of 1:40 000-1:50000. ${ }^{45}$ Non-neurological lesions, which contribute significantly to the morbidity and mortality of affected patients, include angiomatosis of the retina, renal cancer and phaeochromocytoma. The classic manifestations of HLS in the CNS are haemangioblastomas ${ }^{3}$ most of which are located in the posterior fossa but these tumours may also be found in other regions of the CNS. The incidence of haemangioblastoma was $21 \%, 53 \%, 57 \%$ and $72 \%$ respectively, reported in the previously published studies on large kindreds ${ }^{67}$ or small series of families with HLS. ${ }^{89}$ In the present series, CNS haemangioblastoma was found in $44 \%$ of the HLS gene carriers.

Interestingly, we have observed several families without a single instance of CNS haemangioblastoma. The genetic basis for the pattern of the manifestations in different kindreds is, at present, unknown. ${ }^{4}$

Haemangioblastomas of the CNS are of prognostic significance, as they tend to induce the formation of expanding cysts .which may cause life-threatening complications and often require emergency treatment. Cerebellar haemangioblastomas were the major cause of death in $82 \%$ of the patients in the present series; similar figures have been reported by others. ${ }^{7}$ This manifestation is, therefore, of critical importance for the clinical management of HLS patients. Early detection may improve the prognosis of affected patients significantly. To identify asymptomatic carriers and early lesions, we have introduced a standard screening program for patients at risk for HLS (table 1). In addition, genetic linkage analysis with RFLP probes covering the HLS locus on chromosome $3 p$ will provide an opportunity for presymptomatic diagnosis of affected individuals. ${ }^{10} 11$ Screening for CNS lesions should start at the age of 10 years, as our youngest patient with haemangioblastoma was 12 and the youngest patient reported in literature was only 11 years old. ${ }^{9}$

Modern imaging techniques such as MRI scanning and gadolinium-EDTA enhanced MRI (fig 1) have considerably improved the diagnostic repertoire for early detection of small CNS lesions ${ }^{12}$ and for the demonstration of multiple haemangioblastomas, a feature 
encountered in $42 \%$ of our patients and in $50 \%$ in a recently published study ${ }^{9}$ on HLSassociated haemangioblastomas.

The development of multifocal CNS haemangioblastomas appears to be a distinctive feature of HLS, as there was no multiplicity in 51 instances of sporadic intracranial haemangioblastoma. Age was also an important factor: HLS patients with CNS haemangioblastoma became symptomatic about 15 years earlier than patients with sporadic haemangioblastoma. ${ }^{13}$ Other parameters, including location of the tumours and gross or histopathological appearance, were not found to be significantly different between the two groups. We have previously evaluated a series of 63 consecutive CNS haemangioblastomas treated at this institute, $19 \%$ of which were found to be associated with HLS. ${ }^{4}$ Additional manifestations of HLS should be excluded, therefore, in every patient presenting with intracranial or spinal haemangioblastoma.

Follow-up examinations however, are required in these patients. In our series, $21 \%$ had not yet developed additional manifestations of the syndrome at the time of neurosurgical intervention. In $47 \%$ of the HLS patients with haemangioblastoma, non-neurological lesions were identified only during the screening program outlined in table 1 . This indicates that CNS haemangioblastomas are the initial manifestation of the syndrome in a large fraction of HLS patients.

Other CNS manifestations are uncommon. Metastatic renal cell carcinoma was found in the spinal cord of one patient (fig 2). Since both CNS haemangioblastoma and renal cancer represent common features of HLS, the distinction between them can pose a problem. Difficulties may also be encountered in the histopathological differentiation of cerebellar haemangioblastoma and clear cell carcinoma of the kidney. Cytologically, these tumours may be almost indistinguishable. Immunohistochemical reactions with antibodies to epithelial marker antigens, such as cytokeratins or the pan-epithelial antigen Lu-5 may be required to confirm the diagnosis. ${ }^{14}$ In one of our patients, metastatic islet-cell carcinoma of the brain was identified at necropsy. This lesion has only occasionally been described in HLS patients. $^{781516}$

Three members of HLS kindreds of our study presented with ependymoma, astrocytoma and metastatic carcinoid of the brain. As additional features of HLS could not be demonstrated, however, the association with HLS is uncertain. Reports in the literature of atypical CNS neoplasms in patients at risk of HLS, are extremely rare. ${ }^{181517-19}$ To confirm a potential relationship with HLS, such tumours should be evaluated for loss or inactivation of the HLS tumour suppressor gene as soon as the gene is cloned and appropriate reagents become available. ${ }^{2021}$
In conclusion, CNS haemangioblastomas constitute a major manifestation of HLS, are found in about $50 \%$ of gene carriers, tend to develop 15 years earlier compared with sporadic haemangioblastoma, and have a striking tendency for multiple occurrence in HLS patients. Although microsurgical techniques have considerably improved the postoperative outcome in patients with CNS haemangioblastoma, multifocal tumour development and recurrence are still serious problems. Whether novel therapeutic strategies, such as selective embolisation of feeding vessels, can overcome some of these problems remains to be determined.

1 Melmon KL, Rosen SW. Lindau's disease: Review of the literature and study of a large kindred. $\mathrm{Am} \mathcal{f} \mathrm{Med}$ literature and study

2 Neumann HPH. Basic criteria for clinical diagnosis and genetic counselling in von Hippel-Lindau syndrome. $\mathcal{f}$ Vasc Dis 1987;16:220-6.

3 Lindau A. Studien über Kleinhirnzysten. Bau, Pathogenese und Beziehungen zur Angiomatosis retinae. Acta Pathol Microbiol Scand, Supp I, 1926

4 Neumann HPH, Wiestler OD. Clustering of features of von Hippel-Lindau syndrome: evidence for a complex genetic locus. Lancet 1991;337:1052-4.

5 Maher ER. Genetic mechanisms in von Hippel-Lindau disease. Lancet 1991;337:1478-9.

6 Green JS, Bowmer MI, Johnson GJ. Von Hippel-Lindau disease in a Newfoundland kindred. Can Med Assoc $\mathcal{f}$ disease in a Newfour

7 Lamiell JM, Salazar FG, Hsia YE. Von Hippel-Lindau disease affecting 43 members of a single kindred. Medicine 1989;68:1-29.

8 Horton WA, Wong V, Eldridge R. Von Hippel-Lindau disease. Clinical and pathological manifestations in nine families with 50 affected members. Arch Int Med 1976;136:769-77.

9 Filling-Katz MR, Choyke PL, Oldfield E, et al. Central nervous system involvement in von Hippel-Lindau disease. Neurology 1991;41:41-6.

10 Seizinger BR, Rouleau GA, Ozelius LJ, et al. Von HippelLindau disease maps to the region of chromosome 3 associated with renal cell carcinoma. Nature 1988; 332:268-9.

11 Seizinger BR, Smith DI, Filling-Katz MR, et al. Genetic flanking markers refine diagnostic criteria and provide insights into the genetics of von Hippel-Lindau disease. insights into the genetics of von Hippel-1
Proc Natl Acad Sci USA 1991;88:2864-8.

12 Filling-Katz MR, Choyke PL, Patronas NJ, et al. Radiologic screening for von Hippel-Lindau disease: the role of enhanced MRI in the central nervous system. $f$ Comput Assist Tomogr 1989;13:743-55.

13 Neumann HPH, Eggert HR, Weigel $K$, Friedburg $H$, Wiestler OD, Schollmeyer P. Hemangioblastomas of the central nervous system: a ten year study with special reference to von Hippel-Lindau syndrome. 7 Neurosurg 1989;70:24-30.

14 Clelland CA, Treip CS. Histological differentiation of metastatic renal cell carcinoma in the cerebellum from cerebellar hemangioblastoma in von Hippel-Lindau's disease. F Neurol Neurosurg Psychiatry 1989;52:162-6.

15 Cornish D, Pont A, Minor D, Coomles J, Bennington J. Metastatic Islet cell tumor in von Hippel-Lindau disease. Metastatic Islet cell tumor

16 Neumann HPH, Dinkel E, Brambs H, et al. Pancreatic lesions in the von Hippel-Lindau syndrome. Gastroenterology 1991;101:465-71.

17 Kadir S, Kerr WS, Athanasonlis CA. The role of arteriography in the management of renal cell carcinoma with von Hippel-Lindau disease. $\mathcal{F}$ Urol 1981;126:316-9.

18 Lauritsen JG. Lindau's disease, a study of one family through six generations. Acta Chir Scand 1973; 139:482-6.

19 Browne TR, Adams RD, Robertson GH. Hemangioblastoma of the spinal cord. Review and report of five cases. Arch Neurol 1976;33:435-41.

20 Decker HJH, Gemmill RM, Neumann HPH, Walter TA, Sandberg AA. Loss of heterozygosity on $3 p$ in a von Sandberg AA. Loss of heterozygosity on $3 \mathrm{p}$ in a von Het $1989 \cdot 39: 289-93$

21 Tory $\mathrm{KH}$, Brauch $\mathrm{H}$, Linehan $\mathrm{M}$, et al. Specific genetic change in tumors associated with von Hippel-Lindau disease. I Natl Cancer Inst 1989;81:1097-101. 